\title{
Two Elements Elliptical Slot CDRA Array with Corporate Feeding For X-Band Applications
}

\author{
Abdulkareem S. \\ Abdullah \\ Dept. of Electrical \\ Engineering \\ University of Basrah \\ Basrah, Iraq \\ drasabdallah@ieee.org
}

\author{
Asmaa H. Majeed \\ Dept. of Electrical \\ Engineering \\ University of Basrah \\ Basrah, Iraq \\ Asmaahameed37@yahoo.
} co.uk

\author{
Khalil H. Sayidmarie \\ Dept. of Communication \\ Engineering \\ University of Mosul \\ Mousul, Iraq \\ sayidmariek53@uomosul. \\ edu.iq
}

\author{
Raed A. Abd- \\ Alhameed \\ School of Engineering, \\ Design and Technology \\ University of Bradford, \\ Bradford, UK \\ r.a.a.abd@bradford.ac.uk
}

\begin{abstract}
In this paper, a compact two-element cylindrical dielectric resonator antenna (CDRA) array with corporate feeding is proposed for $X$-band applications. The dielectric resonator antenna (DRA) array is excited by a microstrip feeder using an efficient aperture-coupled method. The designed array antenna is analyzed using a CST microwave studio. The fabricated sample of the proposed CDRA antenna array showed bandwidth extending from $10.42 \mathrm{GHz}$ to $12.84 \mathrm{GHz}(20.8 \%)$. The achieved array gain has a maximum of $9.29 \mathrm{~dB}$ i at frequency of $10.7 \mathrm{GHz}$. This is about $2.06 \mathrm{~dB} B_{i}$ enhancement of the gain in comparison with a single pellet CDRA. The size of the whole antenna structure is about $50 \times 50 \mathrm{~mm}^{2}$.
\end{abstract}

Index Terms - CDRA array, microstrip line feeding, elliptical slot.

\section{INTRODUCTION}

The global market of today's communication technology has focused on low profile devices which have become part of the routine life. The ever-increasing drive for miniaturization has led to reduction in the size of the device internal structure as well as the integrated system. The requirements for commercial applications are often quite strict, as the antenna should have compact size, wide bandwidth, high gain, low cost, and be rugged to be widely acceptable. Dielectric Resonator Antennas (DRA) are fabricated from low loss microwave dielectric materials, where the resonant frequency is predominantly a function of size, shape and material permittivity [1-2].

In general, a single element DRA has a broad radiation pattern with limited gain of about $5 \mathrm{~dB}_{\mathrm{i}}$ [3]. Similar to other conventional low gain antennas, DRAs gain can also be enhanced by deploying array configurations. The most attractive and high gain DRA arrays have been introduced in [4-5]. Since last few years, DRA arrays of various shapes like cylindrical, triangular, and rectangular have been proposed in [6-7]. Published works have shown that, the CDRA is the most widely used technology although the rectangular dielectric resonator antenna (RDRA) has shown several advantages. The reason behind that attraction is the simple mode structure of the CDRA as compared to RDRA (larger number of edges in the RDRA lead to more complicated mode [8]. The main advantage of CDRA for high gain applications is that, it is more directional as compared to rectangular and annular shaped DRAs [9]. used to feed linear array of DRAs, such as microstrip lines [10], coplanar waveguide [11], slotted waveguide [12], and dielectric image line [13]. Among these excitation schemes, aperture 
coupling with a microstrip feed line is mainly used because of ease of assembly, suitability to integrate with circuits, and its inherent isolation between the radiating element and feeding network [14].

In a transmitting array, power radiated from each element will impinge on the other elements in the array, while in a receiving array some of the scattered power from each element will impinge upon nearby elements. This interaction between the elements of the array is referred to as mutual coupling. It causes distortions in the desired radiation pattern of the array and can also affect the input impedance of each element, resulting in mismatch losses. The type of antenna element used in the array, its feed network and its design parameters like gain, radiation pattern etc. affect the mutual coupling. The closer the elements placed in the antenna, the higher is the mutual coupling. To find the amount of mutual coupling in an array, the mutual interaction between two elements is often examined first. The element spacing is kept normally from $0.5 \lambda$ to $1.0 \lambda$ to reduce mutual coupling between array elements, and avoid grating lobes. When using DR as array element, the mutual coupling is mostly dependent upon the shape of the DRA, its dielectric constant, and the feed mechanism. The E-plane coupling is stronger than H-plane coupling and it decreases less quickly with increasing element separation [3], [15].

In this paper, a two-element cylindrical DRA array design with corporate feeding network is presented. A slot coupling feeding technique is used, because it is most suitable for DRA arrays where relatively simple circuit integration is required, as compared to other feeding mechanisms. Moreover, the ground plane here isolates the feed network from the radiating $\mathrm{DR}$, thus the radiation characteristics are mainly from the dielectric resonator. The designed antenna is analyzed, and its characteristics such as reflection coefficient, radiation patterns and gain are investigated. The design methodology of the proposed DRA array, based on finite integration technique using a CST microwave studio [16], is deliberated and the detailed results of the proposed antenna are presented.

\section{SINGLE-ELEMENT ANTENNA DESIGN AND PERFORMANCE}

Fig.1 shows the geometry of the proposed single element dielectric resonator antenna fed by an elliptical slot. It is printed on a $0.8 \mathrm{~mm}$ thick Rogers $\mathrm{TMM}^{\mathrm{tm}}$ substrate with relative permittivity $\varepsilon_{r s}=4.5$ and dielectric loss tangent of 0.017 . The ground plane is printed on the substrate with dimensions of $(30 \times 25) \mathrm{mm}^{2}$, which is small enough to meet the circuit boards for many wireless communication applications. The available DRA cylinder has diameter $D=6 \mathrm{~mm}$, height $h=9 \mathrm{~mm}$, and is made of alumina material of $\left(\varepsilon_{r D R}=9.4\right)$. The DRA position is slightly offset from center point of the slot along the feed line. An elliptical shape slot with major slot radius $s_{l}$ and minor slot radius $s_{w}$ is etched on the ground plane as a feeding mechanism for coupling and bandwidth enhancement. Stub length $\left(L_{s t u b}\right)$ was chosen to be as quarter waveguide wavelength. A microstrip feed line with dimensions $L_{f}$ and $W_{f}$ is used to couple the input signal to the DRA through the elliptical slot. $L_{f}$ is chosen as $22.5 \mathrm{~mm}$ to be comparable to the waveguide wavelength, and $W_{f}$ is calculated as $1.5 \mathrm{~mm}$ corresponding to $50 \Omega$, dielectric constant $\left(\varepsilon_{r s}\right)$ and patch thickness $\mathrm{t}$ by using the following standard formula [17]:

$\frac{W_{f}}{t}=\left\{\begin{array}{cc}\frac{8 e^{A}}{e^{2 A}-2} & \text { for } W_{f} / t<2 \\ \frac{2}{\pi}\left[B-1-\ln (2 B-1)+\frac{\varepsilon_{r s}-1}{2 \varepsilon_{r s}}\left\{\ln (B-1)-0.39-\frac{0.6}{\varepsilon_{r s}}\right\}\right] & \text { for } W_{f} / t>2\end{array}\right.$

where,

$$
A=\frac{Z_{o}}{60} \sqrt{\frac{\varepsilon_{r s}+1}{2}}+\frac{\varepsilon_{r s}-1}{\varepsilon_{r s}+1}\left(0.23+\frac{0.11}{\varepsilon_{r s}}\right)
$$

and,

$$
B=\frac{377 \pi}{2 Z_{o} \sqrt{\varepsilon_{r s}}}
$$

The microstrip line is connected to a $50 \Omega$ coaxial SMA connector as a feeding port. The proposed antenna is designed and optimized in terms of its dimension parameters, and the optimized values of parameters are shown in Table I. The resonance frequency for the $\mathrm{TM}_{110}$ (Dominant Mode) can be found from [18]: 
$f_{r_{T M 110}}=\frac{c}{2 \pi a \sqrt{\varepsilon_{r D R}}} \sqrt{(1.841)^{2}+\left(\frac{\pi a}{2 h}\right)}$

where $\mathrm{a}$ and $\mathrm{h}$ are the radius and height of the dielectric resonator respectively. For the used $\mathrm{DR}$, the calculated frequency was found as $10.269 \mathrm{GHz}$.

The realized reflection coefficient and gain of the elliptical slot CDRA are shown in Figs. 2 and 3 respectively. The antenna achieves $-10 \mathrm{~dB}$ bandwidth extending from $9.97 \mathrm{GHz}$ to $12.558 \mathrm{GHz}$ which constitutes a $23 \%$. The antenna gain ranges between $5.5 \mathrm{~dB}_{\mathrm{i}}$ and $7.25 \mathrm{~dB}_{\mathrm{i}}$. The experimental results are also drawn on the same figures. The differences between the measured and simulated results, seen in a shift to higher frequency and general increase in reflection coefficient may be attributed to the fabrication errors and the effects of using glue to fix the DRA.

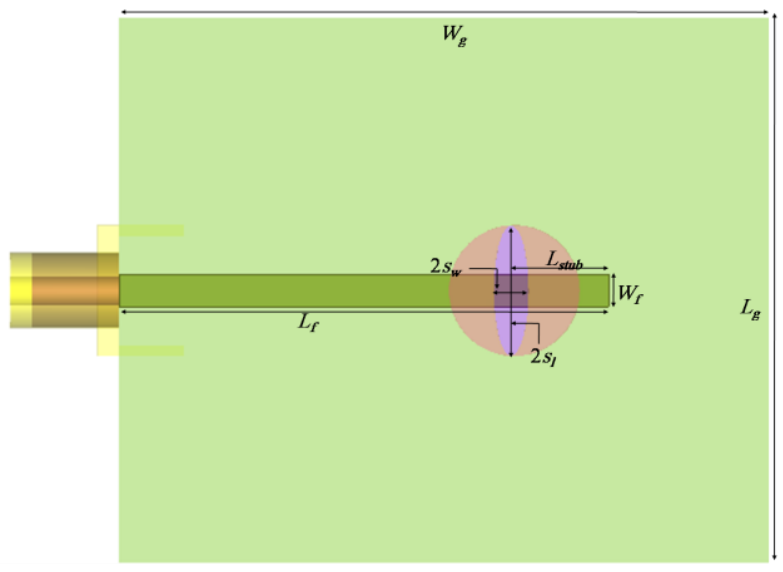

(a)

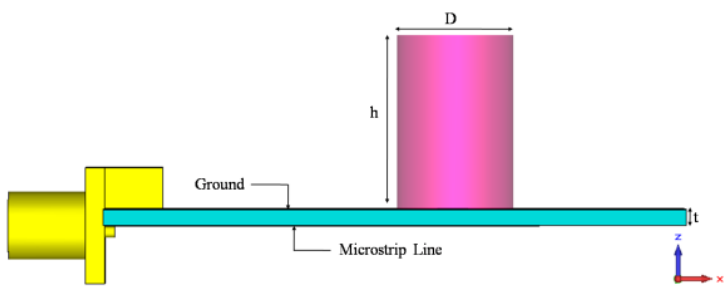

(b)

Fig.1: Elliptical slot cylindrical shape DRA (a)

Top view and (b) Side view with design parameters.
TABLE I

Design dimensions (in $\mathrm{mm}$ ) of the proposed antenna

\begin{tabular}{|c|c|c|c|c|c|}
\hline$L_{g}$ & $W_{g}$ & $D$ & $h$ & $s_{l}$ & $s_{w}$ \\
\hline 25 & 30 & 6 & 9 & 2.98 & 0.8 \\
\hline$L_{\text {stub }}$ & $L_{f}$ & $W_{f}$ & $t$ & \multicolumn{3}{|l}{} \\
\cline { 1 - 3 } 4.5 & 22.5 & 1.5 & 0.8 & \multicolumn{2}{|l}{} \\
\cline { 1 - 3 } & &
\end{tabular}

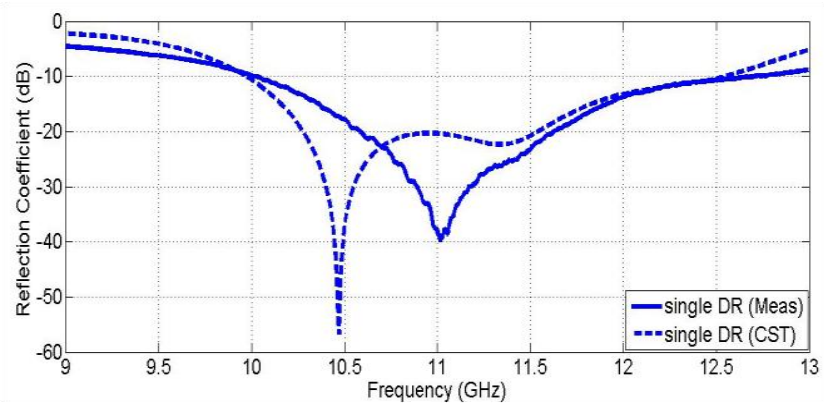

Fig. 2 Simulated and measured reflection coefficient versus frequency for the proposed CDRA.

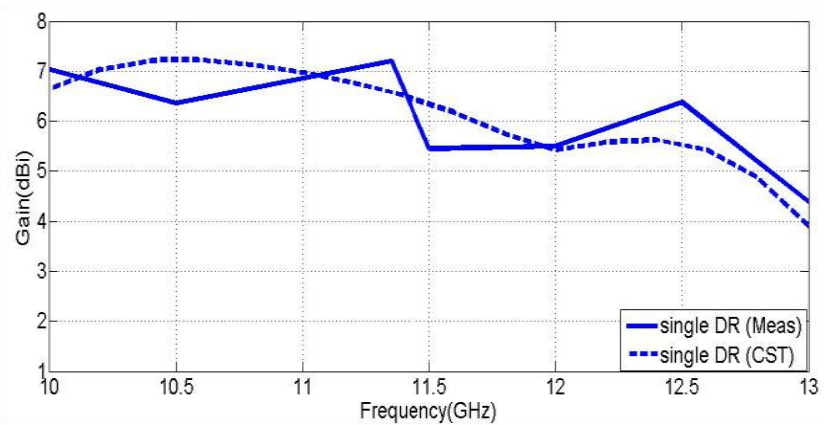

Fig. 3 Simulated and measured realized gain versus frequency for the proposed CDRA.

\section{TWO-ELEMENT CDRA ARRAY USING COOPERATE FEEDING}

The CDR antenna designed in the previous section is used here to form a two-element CDRA array with corporate feed network as shown in Fig.4. The purpose is to maintain the bandwidth and achieve the array response. The array is designed on a ground plane of dimensions $50 \times 50 \mathrm{~mm}^{2}$. This antenna is designed on the basis of using spatial diversity in which the same data is transmitted over each of the multiple paths. In this case each of the two elements is essentially transmitting and receiving the same data although the data is coded differentially.

The optimum distance between two radiating elements (DRs) is determined from simulations to achieve the best reflection coefficient response 
$\left(\mathrm{S}_{11}<-10 \mathrm{~dB}\right)$. The best distance obtained between the two centers of the DRs is $22 \mathrm{~mm}(0.806 \lambda \mathrm{o})$ at a resonance frequency of $11 \mathrm{GHz}$, and reaches $\left(0.916 \lambda_{\mathrm{o}}\right)$ at the highest frequency of operation $(12.5 \mathrm{GHz})$, so that the grating lobes are avoided.

The corporate feed network is used to feed the DRAs in-phase where a $50 \Omega\left(L_{f}=6.6 \mathrm{~mm}, W_{f}\right.$ $=1.5 \mathrm{~mm}$ ) transmission line is divided in two 100 $\Omega\left(L_{f 3}=10.6 \mathrm{~mm}, W_{f 1}=0.34 \mathrm{~mm}\right)$ lines. Both $L_{f}$ and $L_{f 3}$ are chosen as a result of simulations to achieve the best reflection coefficient response $\left(S_{11}<-10\right.$ $\mathrm{dB})$ and they are comparable with the waveguide wavelength. Two quarter- wave transformers at each end are used to match the impedance of these $100 \Omega$ lines to the $50 \Omega\left(L_{f 2}=22.5 \mathrm{~mm}, W_{f}=\right.$ $1.5 \mathrm{~mm})$ lines. These quarter wave transformers of $70.7 \Omega \quad\left(L_{f 1}=10.2 \mathrm{~mm}, \quad W_{f 2}=0.8 \mathrm{~mm}\right) \quad$ impedance have a width of $0.8 \mathrm{~mm}$. The $50 \Omega$ lines are used to feed the DRAs. $W_{f}, W_{f 1}$ and $W_{f 2}$ are calculated according to (1). The design dimensions of the two-element CDRA array using corporate feed network is shown in Table II. The proposed antenna array was fabricated and its performance was measured with HP8510C vector network analyzer. The performance of the proposed array (reflection coefficient magnitude and gain) is compared with the performance of single-element CDRA and the results are shown in Fig.5 and Fig.6.

\section{TABLE II}

Design dimensions (in $\mathrm{mm}$ ) of the two-element CDRA array using corporate feed network.

\begin{tabular}{|c|c|c|c|c|c|}
\hline$L_{g}$ & $W_{g}$ & $D$ & $h$ & $s_{l}$ & $s_{w}$ \\
\hline 50 & 50 & 6 & 9 & 2.98 & 0.8 \\
\hline$L_{\text {stub }}$ & $L_{f}$ & $L_{f 1}$ & $L_{f 2}$ & $L_{f 3}$ & $W_{f}$ \\
\hline 4.5 & 6.6 & 10.2 & 22.5 & 10.6 & 1.5 \\
\hline$W_{f 1}$ & $W_{f 2}$ & $d$ & \multicolumn{4}{|l}{} \\
\hline 0.34 & 0.8 & 22 & \multicolumn{1}{|l}{} \\
\cline { 1 - 2 } & &
\end{tabular}

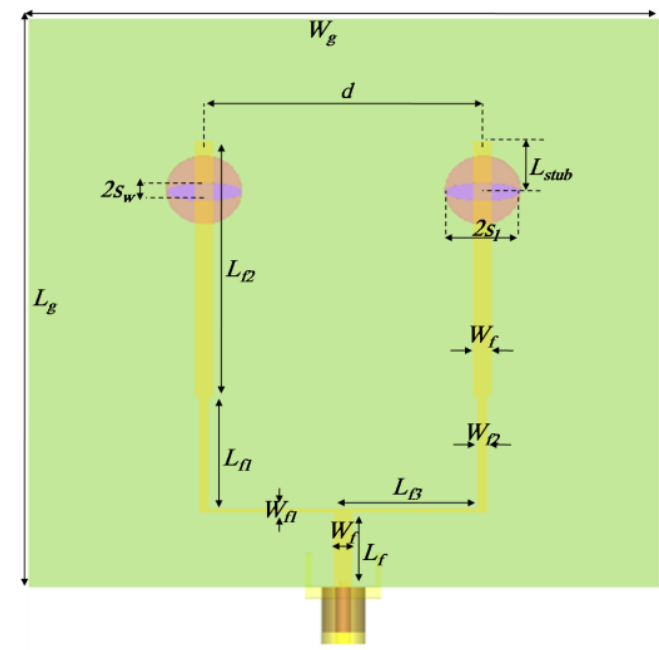

(a)

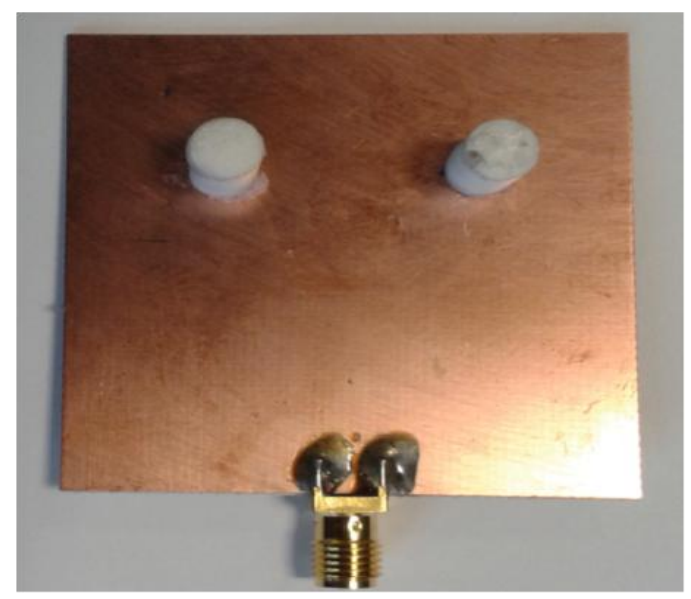

(b)

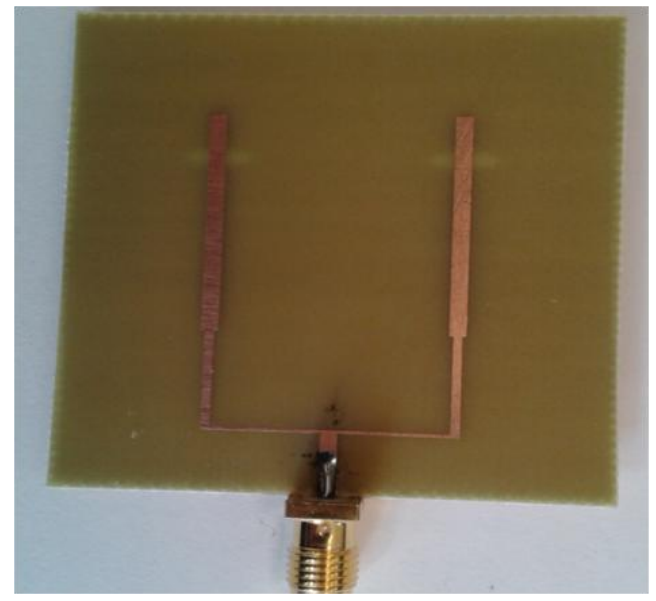

(c)

Fig.4 Two-element CDRA array using corporate feed network; (a) simulated antenna, (b) top view of fabricated antenna and (c) back view of fabricated antenna. 
Fig.5 shows that with single CDR the simulated impedance bandwidth is (9.97-12.558) $\mathrm{GHz}$ with a minimum reflection coefficient of $56.4 \mathrm{~dB}$ at $10.47 \mathrm{GHz}$, whereas it is $(9.936-12.537)$ $\mathrm{GHz}$ with a minimum reflection coefficient of $17.99 \mathrm{~dB}$ at $12.046 \mathrm{GHz}$ using $\mathrm{CDR}$ array. The measured impedance bandwidth with single CDR is $(10.1-12.7) \mathrm{GHz}$ with a minimum reflection coefficient of $-40 \mathrm{~dB}$ at $11 \mathrm{GHz}$, whereas it is $(10.42-12.84) \mathrm{GHz}$ with a minimum reflection coefficient of $-17.80 \mathrm{~dB}$ at $12.07 \mathrm{GHz}$ with CDRA array. The differences between the measured and simulated results, seen in a shift to higher frequency and general increase in reflection coefficient, may be attributed to the effects of the use of glue to fix the DRA, as this has been noticed by [9], and fabrication errors. Moreover, better matching can be usually achieved for a single element as compared to that for the twoelement array with the feed network.

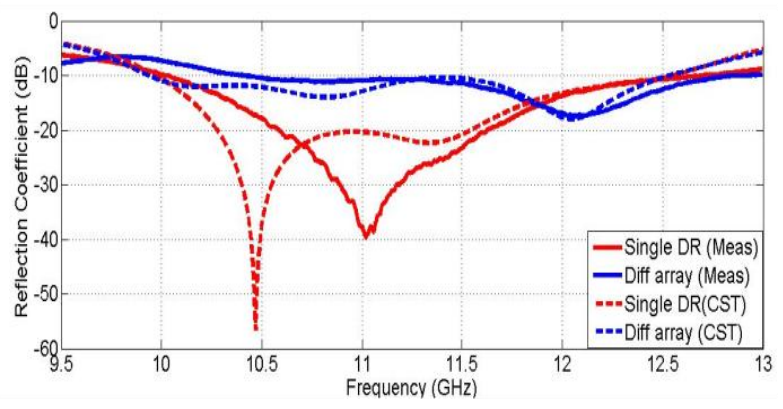

Fig.5 Simulated and measured reflection coefficient curves of the two-element array compared to a single antenna element.

From Fig.6 it is clear that with single CDR the maximum gain is $7.23 \mathrm{dBi}$ whereas using CDR array it is $9.29 \mathrm{dBi}$. Gain improvement of $2.06 \mathrm{dBi}$ is achieved at most of frequencies in the whole frequency band with the CDR array compared to that of a single antenna element. It should be noted that the simulated gain curves assume an ideal feeding network, whereas the measured results include the insertion loss of the used feeding network, hence there are local discrepancies.

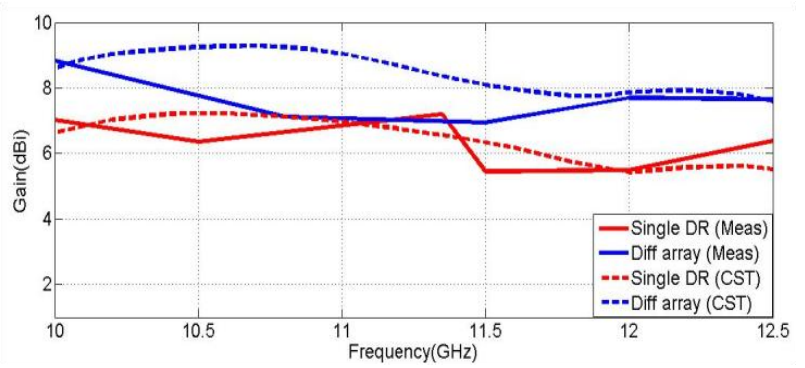

Fig. 6 Simulated and measured gain curves of the two-element array compared to a single antenna element.

A comparison between the simulated far field 3D-radiation patterns of the single-element CDRA and the two-element CDRA array at 10.47GHz and $11.35 \mathrm{GHz}$ are shown in Fig.7a\&b. These figures show the directivity over the phi and theta angles for the two antennas at the considered frequencies. It is clear that for a single-element CDRA the maximum power is radiated in positive $\mathrm{z}$-direction for both 10.47 $\mathrm{GHz}$ and $11.35 \mathrm{GHz}$ frequencies with maximum directivity of $7.29 \mathrm{dBi}$ at $10.47 \mathrm{GHz}$. While the two-element CDRA array produces a constructive superposition increasing the directivity from $7.29 \mathrm{~dB}_{\mathrm{i}}$ to $10.28 \mathrm{~dB}_{\mathrm{i}}$ at $10.47 \mathrm{GHz}$ and from $6.769 \mathrm{~dB}_{\mathrm{i}}$ to $9.684 \mathrm{~dB}_{\mathrm{i}}$ at $11.35 \mathrm{GHz}$. This is due to typical $3 \mathrm{~dB}$ increase due to using two elements. Moreover, the beam is narrower in the YZ-plane due to the array action.

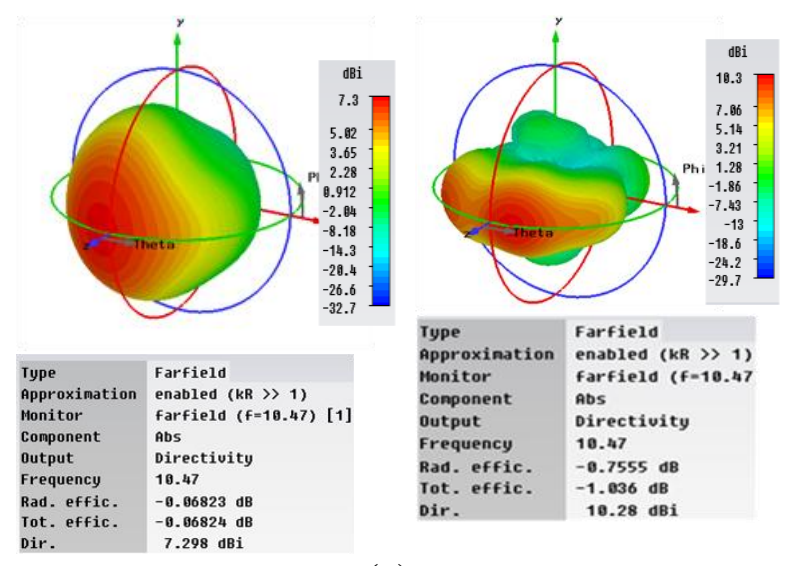

(a) 

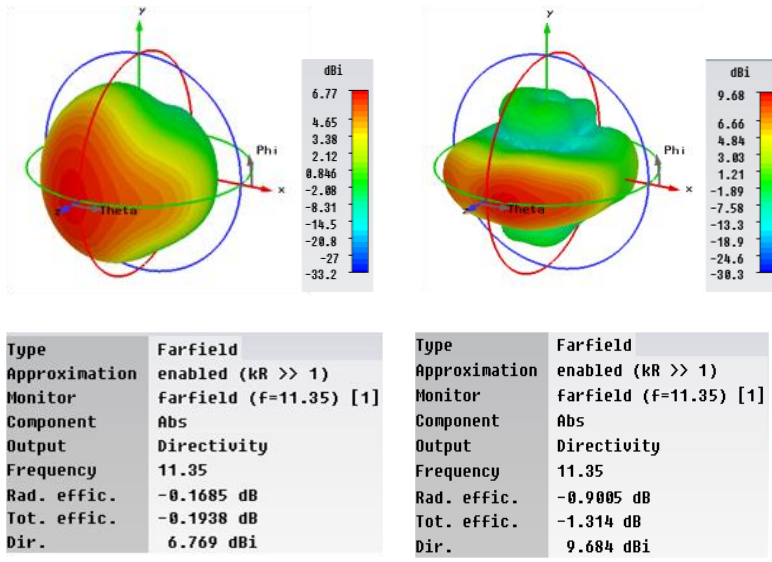

(b)

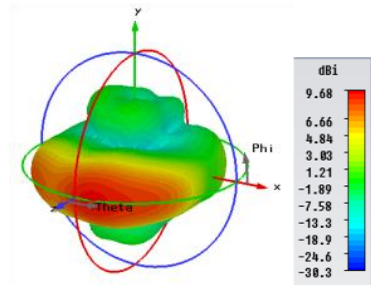

Fig.7 Comparison of the simulated far field 3$D$ radiation patterns for single-element CDRA antenna and two-element CDRA array at; (a)

$10.47 \mathrm{GHz}$ and (b) $11.35 \mathrm{GHz}$.

The simulated and measured far field radiation patterns of the proposed two-element CDRA array are shown in Fig.8. It shows the radiation patterns at three frequencies $(10.1 \mathrm{GHz}$, $10.8 \mathrm{GHz}$ and $12 \mathrm{GHz}$ ). It can be observed that the antenna exhibits a stable radiation behavior across the required operating band. The antenna has low cross-polar response, and wide beam covering more than half space in XZ-plane. In the YZ-plane the pattern has narrow beam, due to array effect, and good front/back ratio is noticed.
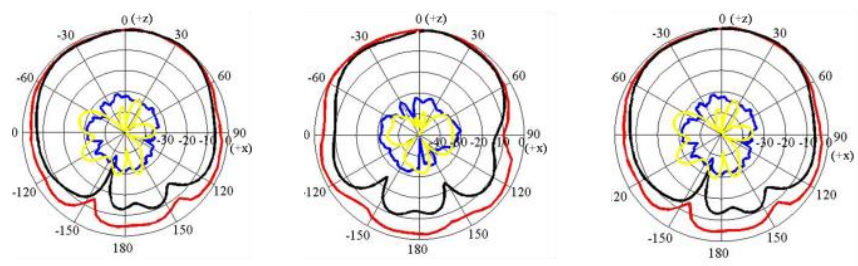

(a)
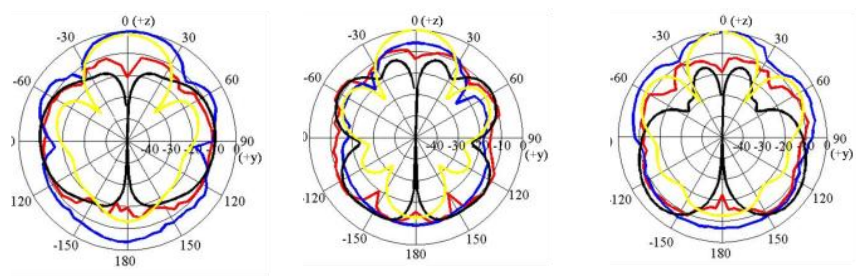

(b)

Fig. 8 Normalized radiation pattern plots of the proposed CDRA array at $(10.1 \mathrm{GHz}, 10.8 \mathrm{GHz}$ and 12GHz) (a) XZ-plane and (b) YZ-plane; $\mathrm{E}_{\theta} \mathrm{CST}$ (black), $\mathrm{E}_{\theta}$ Meas.(red), $\mathrm{E}_{\varphi} \mathrm{CST}$ (yellow) and $\mathrm{E}_{\varphi}$ Meas. (blue).

\section{CONCLUSIONS}

In this paper, a compact two-element cylindrical dielectric resonator antenna array suitable for X-band applications is presented. The proposed CDRA array consists of two cylindrically-shaped dielectric resonators of equal diameters and heights which are excited by corporate feeding network through elliptical slots. The proposed single-element CDRA and the twoelement CDRA array were designed and analyzed. The fabricated samples were tested for performance evaluation. The simulated and measured results showed good agreements, and a gain improvement of $2.06 \mathrm{~dB}_{\mathrm{i}}$ is achieved at most of frequencies in the whole frequency band with the CDRA array compared to that of a single antenna element. A relatively wide band extending from (9.936 to 12.537$) \mathrm{GHz}$ is obtained from simulation results, while the bandwidth extends from (10.42 to 12.84) $\mathrm{GHz}$ for the fabricated sample.

\section{ACKNOWLEDGEMENT}

This work was supported by the Iraqi Ministry of Higher Education and Scientific Research and the Engineering and Physical Sciences Research Council (EPSRC), U.K. under Grant EP/E022936.

\section{REFERENCES}

[1] W. M. Wahab, et al., "Low Cost Planar Waveguide Technology-Based Dielectric Resonator Antenna (DRA) for MillimeterWave Applications: Analysis, Design, and Fabrication," IEEE Transactions on Antennas and Propagation, vol.58, pp.2499-2507, 2010.

[2] A. Agouzoul, et al., "Design of A High Gain Hybrid Dielectric Resonator Antenna for Millimeter-Waves Underground Applications," The IEEE International Symposium on Antennas and Propagation, pp.1688-1691, 2011.

[3] K. M. Luk and K. W. Leung, Dielectric Resonator Antennas, Research Studies Press Ltd., Baldock, Hertfordshire, England, 2003.

[4] L. M. Soo and S. Ho, "A Study on the Enhancement of Gain and Axial Ratio Bandwidth of the Multilayer CP-DRA," 
Proceedings of the 5th European Conference on Circuits and Systems for Communications (ECCSC'10), pp.248-252, 2010.

[5] K. W. Leung, et al., "Two-Dimensional Cylindrical Dielectric Resonator Antenna Array," Electronics Letters, vol.34, pp.12831285, 1998.

[6] R. Kumari, et al., "A Dual Band Triangular Shaped DRA Array for WLAN/WIMAX Applications," The 2011 Annual IEEE in India Conference (INDICON), pp.1-4, 2011.

[7] H. Dashti, M.H. Neshati and F. Mohanna, "RDRA Array Fed by Dielectric Image Line with Improved Gain and Low Back Radiation," $1^{\text {st }}$ International Conference on Communication Engineering, University of Sistan and Baluchistan, pp.41-44, 2010.

[8] D. Yau and M. V. Shuley, "Numerical Analysis of An Aperture Coupled Rectangular Dielectric Resonator Antenna Using A Surface Formulation And The Method Of Moments," IEE Proceedings Microwaves, Antennas and Propagation, Part H, vol.146, pp. 105-110, 1999.

[9] S. Sreekantan, et al., "Simulation and Experimental Investigators on Rectangular, Circular and Cylindrical Dielectric Resonator Antenna," Progress In Electromagnetic Research, vol.7, pp.151-166, 2009.

[10] A. Petosa, et al., "Bandwidth Improvement For A Microstrip-Fed Series Array of Dielectric Resonator Antennas," Electronics Letters, vol.32, no.7, pp.608-609, 1996.

[11] R. Q. Lee, and R. N. Simons, "Bandwidth Enhancement Of Dielectric Resonator Antennas," IEEE Antennas and Propagation Society International Symposium., vol.3, pp.1500-1503, 1993.

[12] I. A. Eshrah, et al., "Theory and Implementation of Dielectric Resonator Antenna Excited by a Waveguide Slot," IEEE Transaction Antennas Propagation., vol.44, no.53, pp.483-494, 2005.

[13] A. S. Al-Zoubi, et al., "Analysis And Design of A Rectangular Dielectric Resonator Antenna FED By Dielectric Image Line Through Narrow Slots," Progress In Electromagnetic Research, vol.77, pp.379390, 2007.
[14] A. H. Majeed, A. S. Abdullah, F. Elmegri, K. H. Sayidmarie, R. A. Abd-Alhameed, J. M. Noras, "Aperture-Coupled Asymmetric Dielectric Resonators Antenna for Wideband Applications," IEEE Antennas and Wireless Propagation Letters, vol.13, pp.927-930, 2014.

[15] D. Guha and Y. M. Antar, "Four-Element Cylindrical Dielectric Resonator Array: Broadband Low Profile Antenna For Mobile Communications," The Proceeding of the XXVIIIth General Assembly of the URSI, New Delhi, India, 2005.

[16] CST Microwave Studio based on the finite integration technique, Framingham, MA, USA, 2011.

[17]D.M. Pozar, "Microwave Engineering," $2^{\text {nd }}$ Edition, John Wiley \& Sons, New York, 1998.

[18] S. A. Long, et al., "The Resonant Cylindrical Dielectric Cavity Antenna," IEEE Transactions on Antennas and Propagation, vol.31, pp.406-412, 1983. 